\title{
IMPORTANCIA DE LA CAPACIDAD DE ALMACENAMIENTO Y USO DE MODELOS LOGÍSTICOS EN EL NIVEL DE PRODUCTIVIDAD DE DISTRIBUIDORAS PYMES DE ARTICULOS DE CONSUMO MASIVO \\ PARA EL HOGAR, 2014
}

\author{
IMPORTANCE OF STORAGE CAPACITY AND LOGISTICS USE OF \\ MODELS IN THE LEVEL OF PRODUCTIVITY OF DISTRIBUTION SMES \\ MASSIVE CONSUMER ITEMS FOR THE HOME
}

\section{MARCOS ESPAÑA GARCÍA', MAYRA CABRERA ARMAS², MÓNICA SÁNCHEZ MOSQUERA ${ }^{3}$}

\author{
1 Universidad de Guayaquil.marcos.espanaga@ug.edu.ec \\ 2 Universidad de Guayaquil.aleja91_-@hotmail.com \\ 3 Universidad de Guayaquil.monikuita91@hotmail.com
}

RESUMEN

El presente trabajo se centra en la evaluación de los problemas que presentan diversas PYMES o distribuidoras del mercado de artículos de uso masivo para el hogar, en sus bodegas. Problemas como la falta de espacio, daños en la mercadería, y la falta de stock afectan directamente su gestión de almacenamiento, nivel de servicio y por ende arriesgan sus niveles de ventas. Debido al constante crecimiento de competidores en este mercado, es necesario plantear propuestas que mejoren sus estrategias de almacenamiento y que reduzca los tiempos de despacho. Y así mejorar el servicio que se brinda a los clientes además de mejorar la eficiencia en los procesos.

PALABRAS CLAVE: mercado, competidores, gestión de almacenamiento, capacidad de bodega, tiempos de despacho, nivel de ventas, procesos.
ABSTRACT

This paper focuses on evaluating the problems presented various SMEs or distribution of mass market items for home use in their cellars. These problems such as: lack of space, damage to the goods, and lack of stock directly affect their storage management, service level and thus risk their sales levels. And due to the steady growth of competitors in this market, you need to make proposals to improve their storage strategies and reduce delivery times. And thus improve the service provided to customers while improving process efficiency.

KEYWORDS: market, competitors, storage management, capacity winery times dispatch, sales level processes. 
INTRODUCCIÓN

Las empresas, dentro de sus operaciones incluyen el manejo de mercaderías, por lo que es necesario que exista un sistema que organice con efectividad este proceso. Ese sistema, se determina modelo de gestión logística y abarca los procesos de almacenamiento, control de inventario y transporte.

Para Bastos (2007) la logística es el proceso por el cual la empresa controla de forma adecuada la distribución y almacenamiento de inventarios y los flujos de información asociados a este. Además un modelo de gestión logística reduce los costos de almacenamiento, ayuda a dar mejor servicio al cliente y a reducir las posibles inversiones de capital.

El problema de varias pequeñas distribuidoras o PYMES del mercado de venta de artículos para el hogar, cuya mayoría se encuentra situada en el centro de la ciudad de Guayaquil; es su poca capacidad de almacenamiento en las bodegas y la deficiencia en el servicio al cliente, por demoras en los tiempos de despacho o por no contar con un modelo logístico adecuado. Por lo tanto, este artículo consiste en un análisis de si la adquisición de bodegas más grandes y la aplicación de un modelo de gestión logística es la solución más factible al problema existente y si aumentará los niveles de productividad de este grupo de PYMES.

ANTECEDENTES

Rivas (2008) con su trabajo "Factibilidad de reubicación de la bodega de producto terminado Durán” mostró que la logística empresarial se ha convertido en un área completamente atractiva para proporcionar un valor agregado a los clientes de las empresas e indicó que la logística debe llegar a convertirse en algún momento en un proceso de flujo continuo, gracias a velocidad de respuesta, formalidad, flexibilidad y sensibilidad de sus entregas. Con relación a esto Parada (2009) mostró que un sistema de control de inventario eficiente aplica métodos de control y análisis en correspondencia con la importancia económica relativa de cada producto. Uno de esos métodos es la clasificación $A B C$ y su aplicación eficiente da paso a la factibilidad práctica en la toma de decisiones de gestión de los inventarios.

Por su parte Arrieta (2011) presentó en su proyecto, "Aspectos a considerar para una buena gestión en los almacenes de las empresas (Centros de Distribución, CEDIS)", que la buena gestión en los centros de distribución depende de las condiciones operativas, administrativas y del conocimiento del tipo de estructura para el almacenamiento que posee la empresa. Indicó que la eficiencia de los mismos se mide mediante la aplicación de indicadores de gestión, que analizan el desempeño de las actividades que se llevan a cabo en los diferentes espacios de la estructura. Finalmente Arrieta (2011) determinó que el estudio, análisis y mejoramiento de las condiciones de almacenamiento de los productos permite a las empresas manejar mejores niveles de servicio al cliente, y un cliente satisfecho lleva a que la empresa, desde su servicio de almacén, tenga un mejor desempeño administrativo y se logren mejores niveles de calidad.

Con relación a esto Maldonado y Villalva (2011) establecen que más que requerimientos de expansión y posicionamiento dentro del mercado, la empresa busca llevar la planificación, el control y rastreo de los materiales dentro de la bodega de producto terminado, para ello se elabora un modelo que parte desde la recepción del producto hasta la entrega final al cliente. Además Maldonado y Villalva (2011) mostraron que el modelo fue elaborado mediante un enfoque logístico y emplearon como herramientas principales el manejo de inventarios, la organización a nivel industrial y la administración de operaciones, obteniendo un modelo que optimizó recursos, tiempos de trabajo y procedimientos, reduciendo errores en los procesos de bodegaje y despacho del producto terminado. Los autores señalaron que el manejo de inventario toma un papel importante dentro de la bodega, por lo que sugirieron que el espacio adecuado de los pasillos internos entre un bloque y otro debe ser de $80 \mathrm{~cm}$; siempre y cuando las etiquetas especifiquen el tipo de producto y que estas sean colocadas en un espacio visible.

Por su parte Castillo y Ortega (2013) enfocaron su proyecto en la reestructuración del diseño de una bodega para mejorar la distribución de sus artículos haciendo uso de la clasificación $\mathrm{ABC}$ de inventario, de un modelo matemático de localización de productos, de un análisis de costo de la inversión y un análisis comparativo de capacidad entre el diseño actual y el diseño propuesto de la bodega; con el fin de facilitar la manipulación y ubicación correcta de los productos y por ende a la reducción de tiempo, para incrementar la rentabilidad. Castillo y Ortega (2013) concluyeron que el diseño propuesto generaba un ahorro del $73.23 \%$ en el costo de almacenamiento de los productos terminados 
en bodega. Con relación a esto Valle y Morales (2014) presentaron un modelo de gestión logística para el manejo de los productos en bodega y el resultado de la aplicación de esos métodos fue la recomendación de la ampliación del espacio físico, porque era el principal causante de la desorganización y problemas que se generaban en los procesos; todo esto con el fin de incrementar los niveles de productividad en la empresa. Esto nos lleva a considerar que la metodología $\mathrm{ABC}$ de clasificación de inventarios es el procedimiento adecuado para la organización de una bodega.

\section{BASES TEÓRICAS DISTRIBUCIÓN DE LA BODEGA}

Vergel, (2011) indica en su análisis de diseño y distribución de una planta, que algunas de las ventajas de tener una buena distribución son:

- Disminución de las distancias a recorrer por los materiales, herramientas y trabajadores.

- Circulación adecuada para el personal, equipos móviles, materiales y productos en elaboración, etc.

- Utilización efectiva del espacio disponible según la necesidad.

- Seguridad del personal y disminución de accidentes.

- Localización de sitios para inspección, que permitan mejorar la calidad del producto.

- Mejoramiento de las condiciones de trabajo.

- Incremento de la productividad y disminución de los costos.

ALMACENAMIENTOS

Las empresas deben de contar un stock suficiente para reducir el efecto de las siguientes amenazas:

- Inflación

- Incremento inesperado de la demanda

- Incumplimiento en el plazo de entrega de los proveedores

MODELO DE GESTIÓN DE INVENTARIOS ABC

Se utiliza para clasificar diversas existencias de una empresa cuando ésta tiene unos inventarios con mucha variedad de productos y no puede destinar el mismo tiempo ni los mismos recursos a cada uno de ellos.

Se clasifica las existencias en tres categorías:
- Existencias A. Son los artículos más importantes, forman aproximadamente el $20 \%$ de los artículos del almacén y, en conjunto, pueden sumar del 60 al 80 $\%$ del valor total de las existencias.

- Existencias B. Son existencias menos relevantes para la empresa que las anteriores. Pueden suponer el $30 \%$ de los artículos del almacén, con un valor de entre el 10 y el $20 \%$.

- Existencias C. Estos representan aproximadamente el $50 \%$ de las existencias de la empresa, pero menos del 5 o $10 \%$ del valor total.

Además de los datos cuantitativos se deben tener en cuenta aspectos como:

- Escasez de suministros,

- Plazos de reposición,

- Caducidad,

- Costo por roturas o daños a las existencias

El valor de consumo anual se calcula con la fórmula:

(Demanda anual) $x$ (coste de artículo por unidad).

La aplicación del sistema de costos $\mathrm{ABC}$ en una empresa para el control de inventarios se empieza por la clasificación de artículos, teniendo en consideración los porcentajes en cantidades físicas y la inversión necesaria. Este sistema nos ayuda a clasificar los inventarios en tres categorías:

- A: Muy importantes.

- B: Medianamente importantes.

- C: Poco o nada importantes, que permitan un control muy fuerte sobre el criterio valor total de las existencias.

CONTROL DE INVENTARIOS

Con un efectivo control de inventario puede determinarse:

- Cuánta mercancía tenemos

- Qué decisiones tomar respecto de la producción o las políticas de liquidación

- Cómo rotar adecuadamente los productos

- Detectar y eliminar el robo de mercancías

- Cuantificar las pérdidas por mercancías dañadas, etc. 
INDICADORES DE GESTIÓN

Anaya (2011) indica que los indicadores de gestión constituyen uno de los instrumentos más importantes para alcanzar los objetivos logísticos que se ha fijado la empresa. Ya que su aplicación representan la base para generar ventajas competitivas sostenibles y posicionarse en el mercado.
Algunos de los indicadores más utilizados son los siguientes:

- Indicadores de Abastecimiento

- Indicadores de inventario

- Indicadores de transporte

- Indicadores para el servicio al cliente

- Indicadores financieros

\section{TABLA 1. INDICADORES DE ALMACENAMIENTO}

\begin{tabular}{|c|c|c|c|}
\hline INDICADOR & DESCRIPCIÓN & FÓRMULA & COMENTARIO \\
\hline $\begin{array}{l}\text { Calidad de pedidos } \\
\text { generados }\end{array}$ & $\begin{array}{l}\text { Número de } \% \text { de pedidos } \\
\text { de compras generadas sin } \\
\text { retraso, } 0 \text { sin necesidades } \\
\text { de información adicional }\end{array}$ & $\frac{\text { Productos generados sin problemas }}{\frac{x 100}{\text { Total de pedidos generados }}}$ & $\begin{array}{l}\text { Costes de los problemas inherentes a la generación errática } \\
\text { de los pedios, como costo de lanzamiento de pedidos } \\
\text { rectificadores, esfuerzo del personal de comrpas para } \\
\text { identificar y resolver problemas, incremento del costo de } \\
\text { mantenimiento de inventarios y pérdidas de ventas }\end{array}$ \\
\hline $\begin{array}{l}\text { Entregas perfectamente } \\
\text { recibidas }\end{array}$ & $\begin{array}{l}\text { Número y \% de pedidos que } \\
\text { no cumplen las especifica- } \\
\text { ciones de calidad servicios } \\
\text { definidas, con desgloce por } \\
\text { proveedor }\end{array}$ & Total de órdenes de compra recibidas & $\begin{array}{l}\text { Costo de recibir pedidos sin cumplir las especificaciones de } \\
\text { calidad y servicio como: costo de retorno, costo de volver } \\
\text { a realizar pedidos, retrasos en la producción, costo de } \\
\text { inspecciones adicionales de calidad }\end{array}$ \\
\hline $\begin{array}{l}\text { Nivel de cumplimiento } \\
\text { de proveedores }\end{array}$ & $\begin{array}{l}\text { Calcula el nivel de } \\
\text { efectividad en las entregas } \\
\text { de mercadería de los } \\
\text { proveedores en la bodega } \\
\text { de productos terminados }\end{array}$ & $\frac{\text { Pedidos recibidos fuera de tiempo } \times 100}{\text { Total pedidos recibidos }}$ & $\begin{array}{l}\text { Identifica el nivel de efectividad de los proveedores de } \\
\text { la empresa y que están afectando el nivel de recepción } \\
\text { oportuna de mercadería en la bodega de almacenamiento, } \\
\text { así como su disponibilidad para despachar a los clientes }\end{array}$ \\
\hline
\end{tabular}

Nota. Indicadores de la logística KPI

\section{TABLA 2. INDICADORES DE INVENTARIOS}

\begin{tabular}{|c|c|c|c|}
\hline INDICADOR & DESCRIPCIÓN & FÓRMULA & COMENTARIO \\
\hline $\begin{array}{l}\text { Índice de rotación de la } \\
\text { mercadería }\end{array}$ & $\begin{array}{l}\text { Proporción entre las ventas y las } \\
\text { existencias promedio. Indica el } \\
\text { número de veces que el capital } \\
\text { invertido se recupera a través de } \\
\text { las ventas }\end{array}$ & $\frac{\text { Costo de venta }}{\text { Inventarios promedio }}$ & $\begin{array}{l}\text { Las políticas de inventario, en general, deben mantener un elevado } \\
\text { índice de rotación, por eso, se requiere diseñar políticas de } \\
\text { entregas muy frecuentes, con tamaños muy pequeños. Para poder } \\
\text { trabajar con este principio es fundamental mantener una excelente } \\
\text { comunicación entre sus empleados y proveedor }\end{array}$ \\
\hline $\begin{array}{l}\text { Índice de duración de } \\
\text { mercadería }\end{array}$ & $\begin{array}{l}\text { Proporción entre el inventario final } \\
\text { y las ventas promedio del último } \\
\text { periodo. Indica cuántas veces dura } \\
\text { el inventario que se tiene }\end{array}$ & $\frac{\text { Inventario final } \times 30 \text { días }}{\text { Ventas promedio }}$ & $\begin{array}{l}\text { Altos niveles en ese indicador muestra demasiados recursos } \\
\text { empleados en inventarios que pueden no tener una materialización } \\
\text { inmediata y que está corriendo con el riesgo de ser perdido o sufrir } \\
\text { obsolescencia }\end{array}$ \\
\hline Exactitud del inventario & $\begin{array}{l}\text { Se determina midiendo el costo de } \\
\text { las referencias que en promedio } \\
\text { presentan irregularidades con } \\
\text { respecto al inventario lógico } \\
\text { valorizado cuando se realiza el } \\
\text { inventario físico }\end{array}$ & $\frac{\text { Valor diferencial S }}{\text { Valor total de inventarios }}$ & $\begin{array}{l}\text { Se toma la diferencia en costos de inventario teórico vs el físico } \\
\text { inventariado, para determinar el nivel de confiabilidad en un } \\
\text { determinado centro de distribución. Se puede hacer también para } \\
\text { exactitud en el número de referencias y unidades almacenadas }\end{array}$ \\
\hline
\end{tabular}

Nota. Indicadores de la logística KPI

\section{TABLA 3. INDICADOR DE TRANSPORTE}

\begin{tabular}{|c|c|c|c|}
\hline INDICADOR & DESCRIPCIÓN & FÓRMULA & COMENTARIO \\
\hline $\begin{array}{l}\text { Comparativo del } \\
\text { transporte (rentabilidad } \\
\text { vs gasto) }\end{array}$ & $\begin{array}{l}\text { Medir el costo unitario de } \\
\text { transportar una unidad, respecto } \\
\text { al ofrecido por los transportadores } \\
\text { en el medio }\end{array}$ & $\begin{array}{l}\text { Costo transporte propio por unidad } \\
\text { Costo de contratar transporte por unidad }\end{array}$ & $\begin{array}{l}\text { Sirve para tomar decisiones acerca de contratar } \\
\text { el transporte de mercaderías o asumir la } \\
\text { distribución directa del mismo }\end{array}$ \\
\hline $\begin{array}{l}\text { Nivel de utilización de } \\
\text { los camiones }\end{array}$ & $\begin{array}{l}\text { Consiste en determinar la } \\
\text { capacidad real de los camiones, } \\
\text { respecto a su capacidad instalada } \\
\text { en volumen y peso }\end{array}$ & $\begin{array}{l}\text { Capacidad real utilizada } \\
\text { Capacidad real camión (kg mt3) }\end{array}$ & $\begin{array}{l}\text { Sirve para conocer el nivel de utilización real de } \\
\text { los camiones y así determinar la necesidad de } \\
\text { optimizar la capacidad instalada y/o evaluar la } \\
\text { necesidad de contratar transporte }\end{array}$ \\
\hline
\end{tabular}


TABLA 4. INDICADORES DE SERVICIO AL CLIENTE

\begin{tabular}{|c|c|c|c|}
\hline INDICADOR & DESCRIPCIÓN & FÓRMULA & COMENTARIO \\
\hline $\begin{array}{l}\text { Nivel de cumplimiento } \\
\text { de entregas a clientes }\end{array}$ & $\begin{array}{l}\text { Consiste en calcular el } \\
\% \text { real de las entregas } \\
\text { oportunas y efectivas a } \\
\text { los clientes }\end{array}$ & $\frac{\text { Total de pedidos NO entregados a tiempo }}{\text { Inventarios promedio }}$ & $\begin{array}{l}\text { Sirve para controlar los errores que se presentan en la } \\
\text { empresa y que no permiten entregar los pedidos a los } \\
\text { clientes. Sin duda, esta situación impacta fuertemente al } \\
\text { servicio al cliente y el recaudo de cartera }\end{array}$ \\
\hline Calidad de facturación & $\begin{array}{l}\text { Número y \% de factura } \\
\text { con error por cliente, y } \\
\text { agregación de los mismos }\end{array}$ & $\frac{\text { Facturas emitidas por errores }}{\text { Total de facturas emitidas }}$ & $\begin{array}{l}\text { Generación de retrasos en los cobros e imagen de mal } \\
\text { servicio al cliente, con la consiguiente pérdida de ventas }\end{array}$ \\
\hline $\begin{array}{l}\text { Causales de ntoas de } \\
\text { crédito }\end{array}$ & $\begin{array}{l}\text { Consiste en calcular el \% } \\
\text { real de las facturas con } \\
\text { problemas }\end{array}$ & $\frac{\text { Total Notas de crédito }}{\text { Total facturas generadas }}$ & $\begin{array}{l}\text { Sirve para controlar los errores que se presentan en la } \\
\text { empresa por errores en la generación de la facturación de } \\
\text { la empresa y qeu inciden negativamente en las finanzas y la } \\
\text { reputación de la misma }\end{array}$ \\
\hline Pendientes por facturar & $\begin{array}{l}\text { Consiste en calcular el } \\
\text { número de pedidos no } \\
\text { facturados dentro del } \\
\text { total de facturas }\end{array}$ & $\frac{\text { Total pedidos pendientes por facturar }}{\text { Total pedidos facturados }}$ & $\begin{array}{l}\text { Se utiliza para medir el impacto del valor de los pendientes } \\
\text { por facturar y su incidencia en las finanzas de la empresa }\end{array}$ \\
\hline
\end{tabular}

Nota. Indicadores de la logística KPI

TABLA 5. INDICADORES FINANCIEROS

\begin{tabular}{|c|c|c|c|}
\hline INDICADOR & DESCRIPCIÓN & FÓRMULA & COMENTARIO \\
\hline Costos logísticos & $\begin{array}{l}\text { Esta pensado para } \\
\text { controlar los gastos } \\
\text { logísticos en la empresa } \\
\text { y medir el nivel de } \\
\text { contribución en la } \\
\text { rentabilidad de la misma }\end{array}$ & $\frac{\text { Costo totales logísticos }}{\text { Ventas totales de la compañía }}$ & $\begin{array}{l}\text { Los costos logísticos representan un \% significativo de las } \\
\text { ventas totales, margen bruto y los costos totales de las } \\
\text { empresas, por ello, deben controlarse permanentemente. } \\
\text { Siendo el transporte el que demanda mayor interés. }\end{array}$ \\
\hline $\begin{array}{l}\text { Márgenes de } \\
\text { contribución }\end{array}$ & $\begin{array}{l}\text { Consiste en calcular el } \\
\% \text { real de los márgenes } \\
\text { de rentabilidad de cada } \\
\text { referencia o grupo de } \\
\text { productos }\end{array}$ & $\begin{array}{l}\text { Venta real producto } \\
\text { Costo real directo producto }\end{array}$ & $\begin{array}{l}\text { Sirve para controlar y medir el nivel de rentabilidad y así } \\
\text { tomar correctivos a tiempo sobre el comportamiento de } \\
\text { cada referencia y su impacto financiero en la empresa }\end{array}$ \\
\hline Ventas perdidas & $\begin{array}{l}\text { Consiste en determinar } \\
\text { el } \% \text { del costo de las } \\
\text { ventas perdidas dentro } \\
\text { del total de las ventas de } \\
\text { la empresa }\end{array}$ & $\frac{\text { Valor pedidos no entregados }}{\text { Total ventas compañía }}$ & $\begin{array}{l}\text { Se controlan las ventas perdidas por la compañía al } \\
\text { no entregar oportunamente a los clientes los pedidos } \\
\text { generados. De esta manera se mide el impacto de la } \\
\text { reducción de las ventas por esta causa }\end{array}$ \\
\hline $\begin{array}{l}\text { Costo por cada } 100 \\
\text { pesos despachados }\end{array}$ & $\begin{array}{l}\text { De cada } 100 \text { pesos que } \\
\text { se despachan, que \% es } \\
\text { atribuido a los gastos de } \\
\text { operación }\end{array}$ & $\frac{\text { Costos operativos bodegas }}{\text { Costo de las ventas }}$ & $\begin{array}{l}\text { Sirve para costear el \% de los gastos operativos de la } \\
\text { bodega respecto a las ventas de la empresa }\end{array}$ \\
\hline
\end{tabular}

Nota. Indicadores de la logística KPI

METODOLOGÍA

El método de investigación a utilizar es el hipotético-deductivo porque parte de lo general a lo particular, analizando conceptos generales de logística y transporte, gestión de inventario y finanzas y condensándolos a lo particular en la adquisición de una bodega para una empresa específica.

\section{MÉTODO DE RECOPILACIÓN DE INFORMACIÓN}

Desde un enfoque cuantitativo, la información recopilada fue de fuentes primarias y secundarias; para lo cual se utilizaron el método descriptivo y el método empírico.

El método descriptivo abarca la observación directa y la consulta a fuentes de parte de otros autores para lograr entender el tema y realizar la exposición narrativa y numérica del mismo.
Dentro del método empírico, se aplicaron las entrevistas a los empleados sobre las actividades que se realizan en la empresa, para determinar la situación actual.

\section{RESULTADOS}

Primero se debe determinar la posición en el mercado en el que se encuentra la PYME frente a sus competidores, y los problemas que la misma tiene de manera interna y externa. Para explicarlo, se tomó el caso de la Distribuidora Plásticos Gacela, ubicada en el centro de la ciudad y dedicada a la venta de artículos plásticos, de loza y aluminio para el hogar.

Se realizaron visitas a las instalaciones de la PYME, y a través de entrevistas, revisión de archivos y observación directa se realizó un aná- 
TABLA 6. MATRIZ FODA

\begin{tabular}{|c|c|c|}
\hline & FORTALEZAS & DEBILIDADES \\
\hline $\begin{array}{l}\text { FACTORES } \\
\text { EXTERNOS }\end{array}$ & $\begin{array}{l}\text { - Experiencia } \\
\text { - Ubicación en zona comercial } \\
\text { - Productos de excelente calidad } \\
\text { - Precios competitivos y accesibles al consumidor } \\
\text { - Variedad de proveedores }\end{array}$ & $\begin{array}{l}\text { - Personal con educación básica } \\
\text { - Atrasos en importación } \\
\text { - Reducido espacio de parqueo } \\
\text { - Desorden y poco espacio en bodegas }\end{array}$ \\
\hline OPORTUNIDADES & Fo & DO \\
\hline $\begin{array}{l}\text { \% de descuentos por parte de proveedores } \\
\text { - Facilidad para conseguir nuevos proveedores de } \\
\text { ser necesario } \\
\text { - Proveer productos a negocios pequeños en } \\
\text { surgimiento } \\
\text { - Oportunidad de ampliarse }\end{array}$ & $\begin{array}{l}\text { - Basándose en los años de experiencia obliga } \\
\text { indirectamente a sus proveedores a competir } \\
\text { entre sí y obtener \% de descuentos atractivos } \\
\text { por parte de ellos } \\
\text { - Los precios accesibles son atractivos para los } \\
\text { negocios en surgimiento }\end{array}$ & $\begin{array}{l}\text { - Al no poder importar con facilidad, se puede } \\
\text { conseguir los productos de importadoras de } \\
\text { terceros y volverlos proveedores momentáneos } \\
\text { - Ampliarse atender en la sucursal a sus clientes } \\
\text { de transporte pesado }\end{array}$ \\
\hline AMENAZAS & FA & DA \\
\hline $\begin{array}{l}\text { - Entrada de nuevos competidores } \\
\text { - Estrategias de precios de la competencia } \\
\text { - Restricciones a la importanción } \\
\text { - Situacion económica y política del país }\end{array}$ & $\begin{array}{l}\text { - La experiencia otorga ventaja ante nuevos } \\
\text { competidores } \\
\text { - Establecer alianzas con competidores para } \\
\text { importar juntos } \\
\text { - Buscar nuevos proveedores extranjeros }\end{array}$ & $\begin{array}{l}\text { - Unirse entre importadores para apelar } \\
\text { restricciones }\end{array}$ \\
\hline
\end{tabular}

lisis FODA de la misma, pero enfocándose en el manejo de sus inventarios se determinó que los problemas que presentaba eran los siguientes:

1. La bodega actual de la distribuidora no contaba ni con espacio, equipos de almacenamiento, o perchas adecuadas. No existía área de despacho ni de averías.

2. La bodega estaba distribuida en tres pisos conectados por una escalera angosta. Lo que resultaba incómodo para los empleados, quienes se tomaban gran tiempo en despachar los pedidos.

3. Los productos no estaban distribuidos de acuerdo a la Clasificación ABC, lo que dificultaba el almacenamiento y aumentaba el tiempo de despacho. Se realizó también un análisis de capaci- dad de almacenamiento, comparando el volumen de mercadería almacenado durante la investigación $\left(1067 \mathrm{~m}^{3}\right)$, con la capacidad real de las instalaciones que eran $1008 \mathrm{~m}^{3}$, se determinó que la bodega estaba sobre-ocupada.

4. Existía inseguridad laboral por falta de espacio para movilizarse dentro de la bodega con artículos de gran volumen. Los pasillos son muy estrechos.

5. En el proceso de recepción y almacenamiento se calculó un tiempo aproximado de 4 horas, 05 minutos y 15 segundos. Mientras que el tiempo aproximado del proceso de despacho fue de 1 hora, 55 minutos y 5 segundos. Se determinó que debido a la infraestructura de la bodega, se demora un tiempo considera-
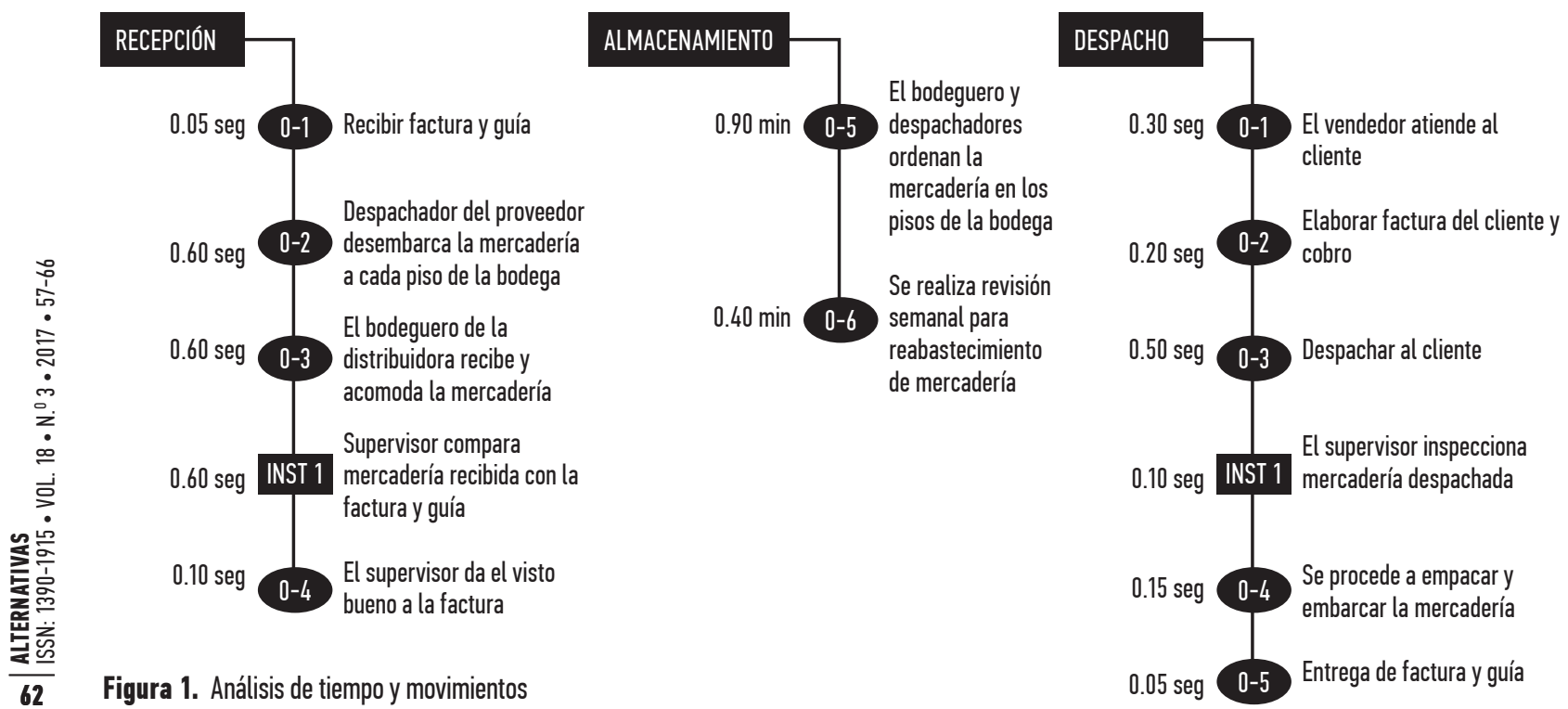

Figura 1. Análisis de tiempo y movimientos 
ble; porque los despachadores realizan varios recorridos para lograr ubicar la mercadería en cada piso de la bodega y de la misma manera para despachar la mercadería vendida.

6. La distribuidora no cuenta con un sistema de control de inventario, por lo que no se sabe con exactitud, si todos los productos rotan de la misma manera y si la empresa se abastece en el tiempo adecuado para cubrir la demanda. Solo se realiza un conteo por observación directa.

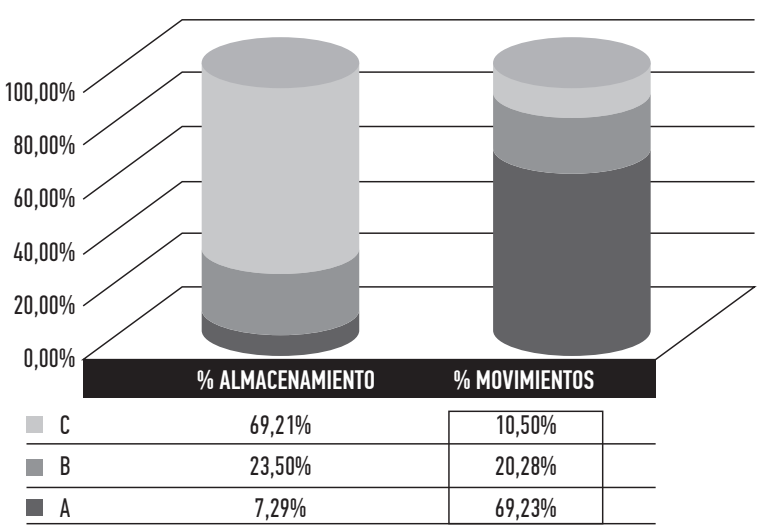

Figura 2. Clasificación $A B C$

La figura 2 nos muestra la clasificación ABC del inventario de la distribuidora en base a la rotación de los productos de Enero - Junio del año 2014. Los artículos de la categoría A tienen un nivel de rotación de $69.23 \%$ que se considera relativamente alto y representa el $7.29 \%$ del inventario almacenado. Los artículos de la categoría B tienen un nivel de rotación de $20.28 \%$ que es equivalente a un nivel medio y representa el $23.50 \%$ del inventario en bodega. Los de lento movimiento con un $10.50 \%$ forman parte de la categoría C, que representan el $69.21 \%$ de los artículos almacenados.

Luego de analizar los problemas, se debe plantear como solución la compra de una nueva bodega de un área de $1470 \mathrm{~m}^{3}$, la cual se distribuye de la siguiente forma:

TABLA 7. CAPACIDAD DE BODEGA

\begin{tabular}{|c|c|c|c|}
\hline ÁREA & MEDIDAS & M3 & \\
\hline Administración & $7 m \times 3 m \times 7 m$ & 147 & \multirow{5}{*}{$1323 \mathrm{~m}$} \\
\hline Almacenamiento & $21 \mathrm{~m} \times 7 \mathrm{~m} \times 7 \mathrm{~m}$ & 1029 & \\
\hline Distribución a futuro & $7 m \times 3 m x 7 m$ & 147 & \\
\hline Montacargas & $2 m \times 3 m \times 7 m$ & 42 & \\
\hline Predespacho & $5 m \times 3 m \times 7 m$ & 105 & \\
\hline Total capacidad de bodega & & 1470 & \\
\hline
\end{tabular}

Como se observa en la tabla 7 el área de uso exclusivo para realizar las operaciones logísticas dentro de la bodega es de $1323 \mathrm{~m}^{3}$. Demostrando así que la capacidad de almacenamiento de la bodega propuesta es mayor a la de la bodega analizada. Además su selección presenta dos beneficios adicionales para la Distribuidora:

- Cercanía de las fuentes de abastecimiento: Tomando en cuenta que los proveedores se encuentran cerca, en el mismo sector, el abastecimiento de la mercadería sería inmediato. La mercadería tendrá menos averías por lo que el trayecto de entrega sería corto.

- Cercanía al mercado (clientes): La empresa podría captar más clientes ubicados en cantones cercanos del Guayas, porque se encontrarían en toda la vía de acceso, evitándoles tráfico y pérdida de tiempo entrando a la zona céntrica de la ciudad. Además algunos de sus clientes propietarios de camiones grandes podrían realizar sus compras en la Distribuidora, sin preocupación de infringir la Ordenanza Municipal, que prohíbe la circulación de estos camiones en el centro de la ciudad durante las horas de la mañana.

Además, las dimensiones más grandes de la nueva bodega, permite crear áreas con funciones específicas para implementar un nuevo modelo logístico. Las áreas son:

- Área de recepción: En esta área se recibirá la mercadería por parte de los proveedores, los cuales fueron clasificados en dos categorías:

- La categoría "A", incluyen a proveedores que se les otorgó un $100 \%$ de confiabilidad por cumplimiento en tiempos de entrega, mercadería completa y sin averías. Sus productos pasan por el andén directo a ser almacenados, y el único control que se aplica es el conteo de los bultos para compararlos con los de la factura recibida.

- La categoría "B", son aquellos que han incumplido en sus tiempos de entrega y han presentado problemas por mercadería incompleta. Y antes de ser almacenados son detenidos en el área de recepción para revisar 
que las cajas no estén abiertas, la cantidad de unidades completas y sin averías. Con esto se evitarán futuros imprevistos al momento de despachar mercadería.

Finalmente para poder categorizar a los proveedores de la empresa, se deben aplicar los siguientes indicadores:

- Entregas perfectamente recibidas:

Pedidos Rechazados $\times 100$

Ordenes de compras recibidas

- Nivel de cumplimiento de proveedores:

Pedidos recibidos fuera de tiempo $x 100$ Total de pedidos recibidos

- Área de almacenamiento: El objetivo de expandir y tener una sola área de almacenamiento es de conseguir un flujo de las mercaderías más rápido dentro de la bodega, que facilite el despacho de los pedidos de manera eficiente y el manejo del inventario, logrando así mejorar el nivel de productividad y de atención al cliente.

Los pasillos van a estar ubicados de forma lineal, serán amplios para permitir el paso de montacargas y procurar que exista la menor cantidad de cruces que obstruyan el acceso a las salidas. La mercadería estará ubicada en las nuevas estanterías de acuerdo a la clasificación $A B C$, en base a su rotación. Las estanterías más cerca de la zona de despacho, contendrá la mercadería de la clasificación A que son los productos de mayor rotación; las siguientes los productos de la categoría B o rotación media y por último las siguientes estanterías los de la categoría C o baja rotación.

El proceso de almacenamiento comienza en el área de recepción, una vez que la mercadería es preparada para su almacenamiento. Y termina cuando el bodeguero reporte al supervisor que la mercadería esté completa.

Para evitar robos internos y faltas de abastecimiento se tomarán medidas de control mediante la realización de tomas físicas de inventario cada dos semanas.
- Área de pre-despacho: En esta área estará ubicada la mercadería despachada por el personal de bodega y que el cliente aún no retira. Debido a que esta mercadería ocupa espacio, se establecerá como política de funcionamiento para esta área que el tiempo máximo que dura la mercadería almacenada sea de una semana; si se excede de este plazo se tomarán las siguientes medidas:

- Se contactará al cliente para indicarle que tiene un plazo de 48 horas para retirar la mercadería, pero se le aplicará un recargo de $\$ 1,00$ por día.

- En caso de no retirarla después de 48 horas, la mercadería pasa nuevamente al área de almacenamiento para usarla en una nueva venta.

Además, se plantea en la propuesta el uso del siguiente indicador para el control de productividad en esta área:

- Nivel de cumplimiento de despacho:

Número de despachos cumplidos $x 100$

Número total de despachos requeridos

- Área despacho: En esta área se cargan los camiones de los clientes cuando llegan a retirar sus compras o el camión propio de la empresa para llevarlas a cooperativas de transporte y enviarla a ciertos clientes.

- Área de administración: El fin de esta área es brindar una atención personalizada tanto a proveedores como a los clientes, donde los primeros podrán hacer la entrega de las facturas y las guías y cobro. Los clientes en esta área podrán realizar sus pedidos. De la misma forma, ambos podrán presentar algún tipo de queja o reclamo, si existiera, dentro de esta área. Para llevar un control sobre la eficiencia del área, se propuso aplicar el siguiente indicador:

- Calidad de Facturación:

Facturas emitidas por errores

Total facturas emitidas

Área Distribución de futuro: En la propuesta se determinó que esta área se conformará por mercadería de temporada o de uso futuro, es decir, artículos que únicamente se van a vender en meses establecidos, como el día de la madre, 
regreso a clases, navidad, entre otras fechas. Esta mercadería se la va adquirir con dos meses anticipadamente y se la va a tener almacenada en esta bodega hasta que se la necesite.

Posterior a la determinación de los problemas de la PYME y presentación de la propuesta, se analizó la viabilidad económica de la solución propuesta. En el caso de la Distribuidora Gacela se realizó la proyección de ingresos y costos, elaboración del Estado de Resultados Proyectados, el cálculo del Flujo de Caja, el Valor Actual Neto (VAN), la Tasa Interna de Retorno (TIR) y el Tiempo de Recuperación de Inversión. Los resultados más relevantes y que muestran la factibilidad económica fueron la TIR de 33,02\% y el VAN positivo de $\$ 162.422,33$

Finalmente en base a los resultados de la evaluación financiera se recomienda la implementación de la propuesta ya que incidirá favorablemente en la productividad de la empresa.

CONCLUSIONES Y RECOMENDACIONES

Del análisis de los problemas y la solución propuesta se obtienen las siguientes conclusiones:

- Ampliar la capacidad de almacenamiento de la bodega permite un mejor almacenamiento. Incluso reduce el tiempo de despacho de los pedidos.

- Con la categorización de los proveedores y aplicación de indicadores del modelo logístico propuesto se logra medir los niveles de productividad; cumpliendo con los tiempos de recepción de mercadería, la atención al cliente, entre otros.

- El almacenamiento y la preparación de pedidos será más ordenado gracias a la aplicación del Sistema ABC.

- Al implementar nuevas medidas de almacenamiento y señalización, aumenta el nivel de seguridad del personal.

- La distribuidora incrementará su nivel de productividad por mejor uso de espacio y mejor tiempo de respuesta al cliente.

- La diferencia en la capacidad de almacenamiento entre la bodega actual y la bodega propuesta es de $315 \mathrm{~m}^{3}$, demostrando así que la capacidad de la nueva permitirá un mejor almacenamiento.

- Con la categorización de los proveedores y aplicación de indicadores del modelo logístico propuesto se logra medir los niveles de rotación; cumpliendo con los tiempos de recepción de mercadería, la atención al cliente, entre otros.

- El almacenamiento y la preparación de pedidos será más ordenado gracias a la aplicación del Sistema ABC. Incluso se reduce el tiempo de despacho de $1 \mathrm{~h}$ $35 \mathrm{~min}$ 05seg.

- Al implementar de las nuevas medidas de almacenamiento y señalización, aumenta el nivel de seguridad del personal.

- La distribuidora incrementará su nivel de productividad en un $31 \%$. Y sus ventas en dólares anuales a un 20\%

- El Estado de Resultados presenta utilidades en todos los años proyectados.

- La TIR del proyecto es de $41.36 \%$

- La propuesta tiene un VAN positivo de $\$ 262.018,10$ lo que confirma la factibilidad de la propuesta.

- La inversión total de $\$ 224.778,46$ se recuperaría a partir es de 3 años y 7 meses.

\section{DISCUSIÓN}

La presente investigación muestra la importancia del análisis ABC para el manejo de inventarios ya que muestra de manera clara los productos de mayor a menor rotación versus las cantidades almacenadas y estas dos características al estar directamente relacionadas, muestran la eficiencia del manejo de los inventarios de una organización. Para este caso en particular el análisis $\mathrm{ABC}$ nos indica una deficiente administración de los niveles de inventario.

Para estudios posteriores se puede ampliar el análisis de inventarios calculando los puntos de reorden para los artículos de mayor rotación en base al análisis ABC.

\section{REFERENCIAS BIBLIOGRÁFICAS}

Arrieta, J. (2011). Aspectos a considerar para una buena gestión en los almacenes de las empresas (Centros de Distribución, CEDIS). (Journal of Economics, Finance and Administrative Scien-

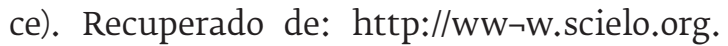
pe/scielo.php?script=sci_arttext\&pid=S207718862011000100007 \&lang $=\mathrm{pt}$

Bastos, A. (2007). Distribución Logística y Comercial. España: Ideaspropias Editorial.

Castillo, R y Ortega, G. (2013). "Optimización en el diseño de una bodega para una empresa que elabora fundas plásticas" (Repositorio de la ESPOL). Recuperado de: http://www.dspace. espol.edu.ec/bitstream/123456789/25042/2/ Trabajo_Final_Graduacion.pdf 
Maldonado, k., y Villalva, M. (2011). Diseño de la gestión logística de la bodega de producto terminado de la división de cartón en Papelera Nacional S.A. (Tesis de Posgrado, Repositorio Digital Universidad Politécnica Ecuatoriana Salesiana). Recuperado de: http://dspace.ups. edu.ec/handle/123456789/2166

Mejía, G y Padilla, P. (2010). Formulación e implementación de un control interno al sistema de compra, percepción, almacenaje, despacho y ventas de mercadería en ferretería Espinoza S.A. según el modelo costo. (Repositorio Institucional, Universidad de Cuenca). Recuperado de: http://dspace.ucuenca.edu.ec/ handle/123456789/3266

Parada, O. (2009). Un enfoque multicriterio para la toma de decisiones en la gestión de inventarios. (Cuad. Adm. vol.22 no.38). Recuperado de: http://search.scielo.org/?q=Un\%20 enfoque $\% 20$ multicriterio $\% 20$ \& where=ORG
Rivas, H. (2008). Factibilidad de reubicación de la bodega de producto terminado Duran. (Biblioteca UTPL). Recuperado de: http://dspace.utpl. edu.ec/handle/123456789/6293

Sapag, N y Sapag, R (2008). Preparación y evaluación de proyectos. Santiago: McGraw-Hill Interamericana de Chile Ltda.

Valle, G. y Morales, E. (2014). Diseño de un modelo de gestión logística en la empresa Megaprofer S.A de la ciudad de Ambato para mejorar los niveles de productividad. (Repositorio PUCESA). Recuperado de: http://repositorio.pucesa. edu.ec/jspui/handle/123456789/932

Vergel, J (2009). Propuesta y Análisis del diseño y distribución de Planta de Alfering Limitada Sede II. Recuperado de http://dspace.universia.net/bitstream/2024/392/1/DISTRIPLANTAFINAL-ALFERING.pdf 